\title{
Solitary, adult-onset, intraosseous myofibroma of the finger: report of a case and review of literature
}

\author{
Yihong Ma • Gene P. Siegal • Shi Wei
}

Published online: 24 December 2014

(C) American Association for Hand Surgery 2014

\begin{abstract}
Myofibroma is a rare benign neoplasm of myofibroblastic origin. It typically occurs in the skin and subcutaneous tissues of the head and neck in infants and young children as multicentric lesions known as infantile myofibromatosis. Intraosseous myofibromas are very rare and are typically destructive lesions that predominantly affect craniofacial bones in the setting of myofibromatosis. Solitary, intraosseous myofibromas in adults are exceedingly rare. Herein, we report a myofibroma involving the middle phalanx of the right index finger in a 58-year-old man who presented with a pathologic fracture. Twelve other cases of adult-onset, intraosseous myofibroma were compiled from the English language literature and integrated with this report.
\end{abstract}

Keyword Myofibroma $\cdot$ Intraosseous · Adult-onset $\cdot$ Solitary

\section{Background}

Myofibroma is a rare benign neoplasm of myofibroblastic origin. It typically occurs in the skin and subcutaneous tissues of the head and neck in infants and young children as multicentric lesions known as infantile myofibromatosis [1], although other anatomic sites may also be rarely affected, including bone and visceral organs. Histologically, the tumor typically exhibits a well-circumscribed, multinodular, biphasic growth pattern characterized by cellular regions consisting of plump spindled cells arranged in a hemangiopericytomalike fashion alternating with less cellular areas composed of more mature myofibroblasts with abundant extracellular matrix. The skeletal involvement is predominantly limited to the

Y. Ma $\cdot$ G. P. Siegal $\cdot$ S. Wei $(\bowtie)$

Department of Pathology, The University of Alabama at

Birmingham, NP 3542, 619 19th St. South, Birmingham,

AL 35249-7331, USA

e-mail:swei@uab.edu craniofacial bones, chiefly in the setting of myofibromatosis [2-4]. Solitary, adult-onset, intraosseous myofibroma is exceedingly rare, with only a handful of case reports. These lesions, once again, mostly occur in gnathic bones. Herein, we report the first case of solitary, adult-onset, intraosseous myofibroma involving the bones of hands and feet. Twelve other cases of adult-onset, intraosseous myofibroma from the English language literature were integrated with this report.

\section{Case report}

The patient, a 58-year-old right-handed man with no significant past medical history, injured his right index finger while mowing his lawn and presented to an emergency room. Conventional radiographs demonstrated a lytic lesion in the middle phalanx of the right index finger with cortical disruption indicating a non-displaced fracture (Fig. 1). He was referred to the authors' institution for definitive management. He denied fever, chills, and unintentional weight loss.

The patient underwent an open biopsy and curettage of the bone tumor followed by grafting. The curetted specimen was composed of fragmented tan-pink soft tissue. Histopathologic examination (Fig. 2a-c) of the lesion revealed a spindle cell neoplasm with a multinodular growth pattern. The lesion exhibited alternating hypercellular and hypocellular zones. While the latter contained plump spindle cells with an eosinophilic cytoplasm along with prominent dystrophic calcifications. The cellular areas were composed of a mixture of spindled and round to polygonal cells arranged in a hemangiopericytoma-like growth pattern. There was mild nuclear atypia in areas. Mitotic figures were not discernable. No necrosis was noted. The lesional cells were immunoreactive for smooth muscle actin (Fig. 2d) but negative for S-100 protein, h-caldesmon, CD34, and broad-spectrum cytokeratin cocktail (data not shown). Thus, the pathologic diagnosis of myofibroma was rendered. The postoperative course was 
Fig. 1 Conventional radiographs demonstrate a well-demarcated lytic lesion with sclerotic rim and a non-displaced fracture
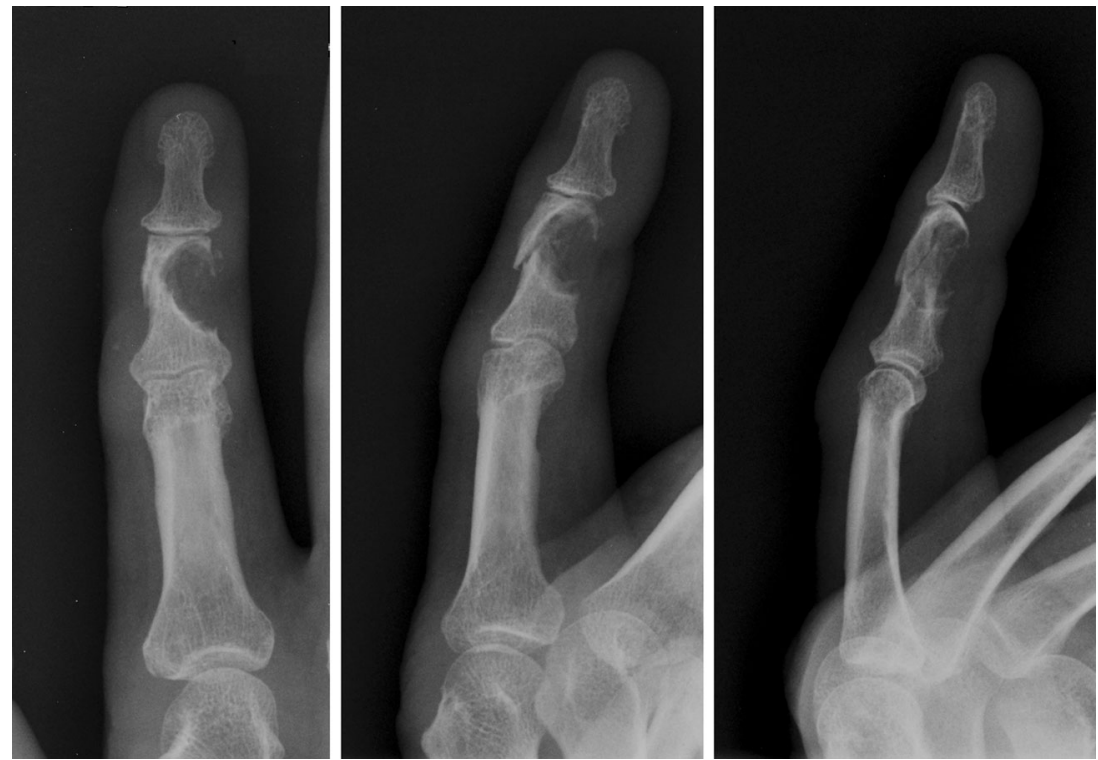

uneventful until 1 year later when he noticed a swelling at the same location. A recurrence was suspected. However, the patient denied further surgical intervention.

\section{Discussion}

Infantile myofibromatosis, formerly known as congenital generalized fibromatosis, was first described by Stout in 1954 [1].
Dermis and subcutis of head and neck regions are the most common locations although other anatomic sites may also be rarely affected, including bone and visceral organs. A subset of solitary and multiple forms of infantile myofibromatosis are familial, with an autosomal-dominant mode of inheritance and variable penetrance. The term of myofibroma was introduced for acquired lesions by Smith et al. in 1989 [4]. The skeletal involvement is predominantly limited to the craniofacial bones. Oudijk et al. analyzed 114 cases of solitary, multifocal, and generalized myofibromas. Eight of 95 (8.4\%) solitary
Fig. 2 Histologic characteristics of myofibroma. The lesion exhibits a multinodular growth pattern with alternating hypercellular and hypocellular areas and dystrophic calcification. Note the reverse zonation with the so-called "myoid nodules" in the center $(\mathbf{a}, \times 40)$. The cellular areas are composed of a mixture of spindled and round/polygonal cells arranged in a

hemangiopericytoma-like growth pattern $(\mathbf{b}, \times 100)$. The myoid cells have bland nuclei and prominent cytoplasmic eosinophilia (c, $\times 400)$. The lesional cells are immunoreactive for smooth muscle action $(\mathbf{d}, \times 100)$
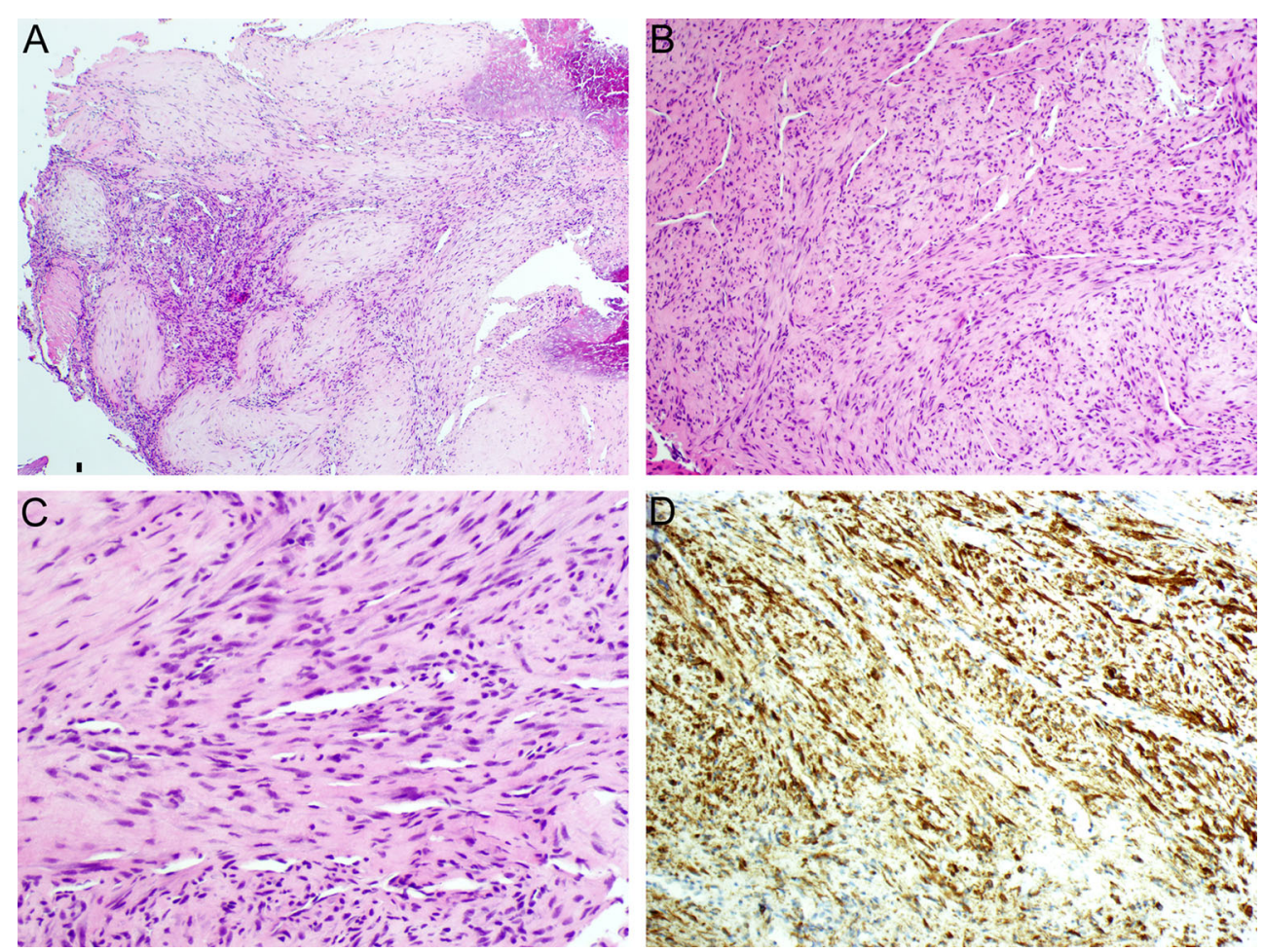
lesions were intraosseous, including three in mandible and two in parietal bone, with others involving clavicle, scapula, and tibia, respectively. Of note, all of the eight patients with intraosseous myofibroma were younger than 25 years of age, of which six were younger than 15 years [3]. Inwards et al. reviewed 14 cases of solitary infantile myofibromatosis of bone and found all but one involving craniofacial bones, with the latter occurring in the tibia. While the age ranged 6 months to 16 years, most of the tumors $(71 \%)$ were in patients aged 2 years or younger [2].

Upon review of the English language literature, a total of 12 acquired/adult-onset (age 15 or older) solitary, intraosseous myofibromas with detailed clinicopathologic characteristics were found and are summarized in Table 1. There were eight men and five women in this cohort including the current case, with an age range of 15 to 58 years. These lesions again predominantly involved craniofacial bones $(54 \%, 7 / 13)$, exclusively affecting mandibles $(n=6)$. Other sites of bone involvement included clavicles, spines, and phalanx. All patients had solitary lesions. Compared to their soft tissue counterpart, intraosseous lesions are insidious when they are small. Patients may present relatively late until unfortunate fractures occur (as in the current case) or until significant bone expansion causing deformity and severe pain. On imaging, the tumors were welldemarcated, radiolucent lesions with variable marginal sclerosis, with or without pathologic fracture. The differential diagnosis is broad and includes other benign lesions such as nerve sheath tumor, fibrous dysplasia, enchondroma, hemangioma, aneurysmal bone cyst, and an infectious process. When they appear as an illdefined radiolucency, more aggressive lesions such as fibrous histiocytoma, fibrosarcoma, Rosai-Dorfman disease, Langerhan cell histiocytosis, desmoid tumor and metastasis are also in the differential diagnostic consideration. Those involving appendicular bones, although extremely rare, tend to occur in the metaphysis, some demonstrating cortical expansion and thus may render a differential diagnosis of metaphyseal cortical defect or chondromyxoid fibroma.

Histologic recognition of this entity in bone is critical since the intraosseous lesions are extremely rare, and their lytic growth pattern is difficult to be distinguished from other lesions radiographically. The microscopic appearance of bony lesions is not much different from its soft tissue counterpart, which typically shows a multinodular, biphasic growth pattern with alternating hypercellular and hypocellular regions, although the two components may be present in variable proportions. The dark-stained, cellular regions consist of densely packed round to spindle-shaped myofibroblasts with scant eosinophilic cytoplasm and indistinct cell borders.
The light-stained, less cellular areas are composed of myofibroblasts with abundant extracellular matrix that often refers to as "myoid nodules." A perivascular growth pattern is often recognizable in the cellular areas surrounding the myoid lobules and, in fact, these lesions were initially thought to lie on a histologic continuum with the other tumor types having a perivascular myoid differentiation, including myopericytoma and glomangiopericytoma. The distinction between them is sometimes arbitrary due to considerable overlap. Stromal hyalinization and calcifications may be present and occasionally dominant the lesion thus complicating the diagnosis. The tumor cells are invariably bland and mitotic figures are rare to absent. The overwhelming majority of myofibromas are immunoreactive for smooth muscle actin but lack expression of other smooth muscle markers (h-caldesmon and desmin), S-100 protein, and $\mathrm{CD} 34[3,5,6]$.

The histologic differential diagnosis for bony tumors largely includes smooth muscle tumors, nerve sheath tumors, solitary fibrous tumor/hemangiopericytoma, benign fibrous histiocytoma, desmoid tumor, and perivascular myoid lesions [1, 2]. Leiomyomas typically have a more homogeneously fascicular growth pattern, with longer spindle cells with cigar-shaped nuclei and brightly eosinophilic cytoplasm, and strongly express other smooth muscle markers such as h-caldesmon and desmin. The biphasic growth pattern of myofibroma may closely resemble a schwannoma. However, the latter characteristically demonstrate fibrillary, cellular areas often arranged in a palisading fashion (Antoni A) admixed with a seemingly distinct loose textured pattern with frequent microcysts (Antoni B). These lesions invariably show strong and diffuse expression of S-100 protein. Solitary fibrous tumor/hemangiopericytoma typically exhibits a patternless architecture with a staghorn branching vascular pattern and perivascular hyalinization. The tumor cells are characteristically immunoreactive with CD34. Benign fibrous histiocytoma can occur in any age and may affect any bone. The lesional cells are typically arranged in a storiform pattern intermixed with variable amount of foam cells and multinucleated giant cells and stain positively with factor XIIIa. Other perivascular myoid lesions usually lack the classic biphasic appearance and myoid nodules of myofibroma. Myopericytoma may also arise in bone and rarely causes oncogenous osteomalacia [7]. Historically, myopericytomas can be separated from myofibroma by their immunoprofilesthe former stains positively with smooth muscle actin and h-caldesmon in contrast to myofibroma, which typically shows no or minimal h-caldesmon immunoreactivity. However, it is now considered by the WHO Working Group that myopericytoma form a morphologic 


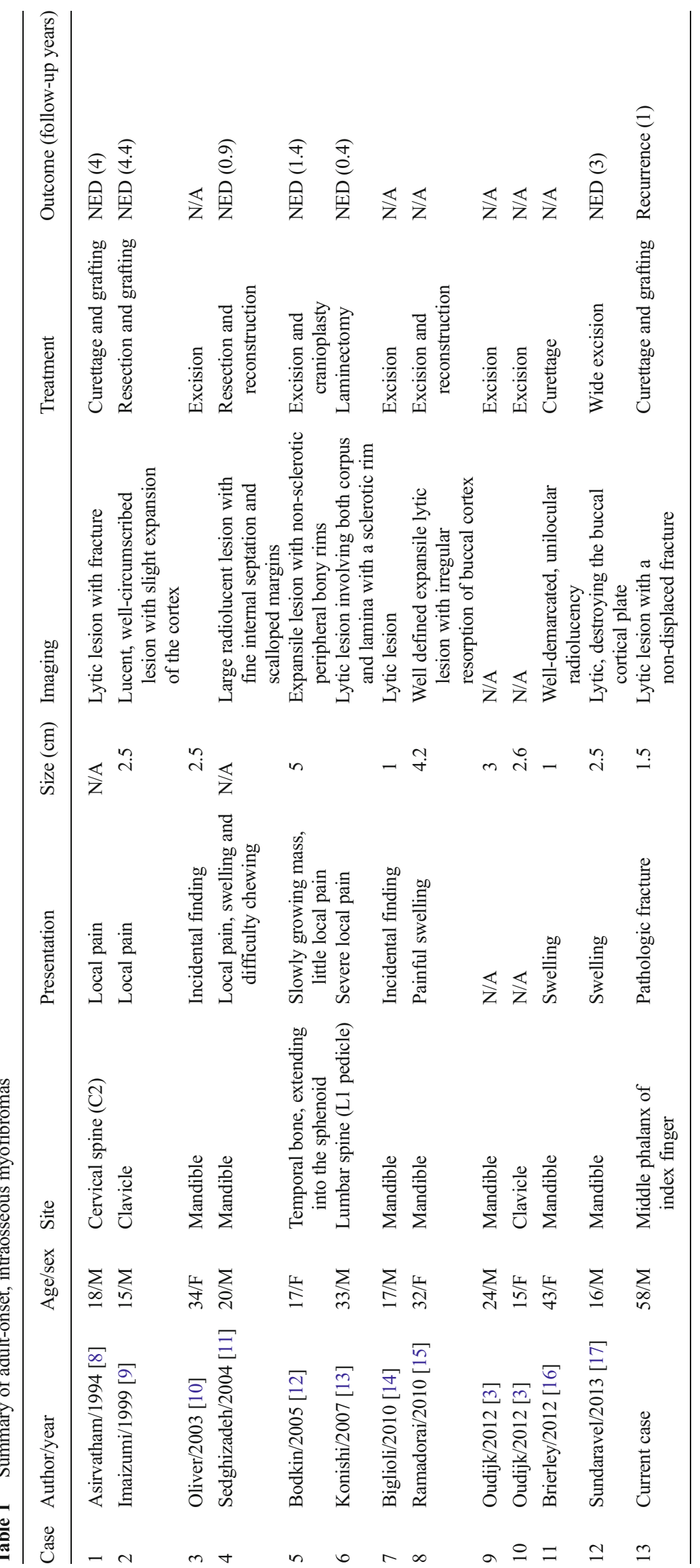


spectrum with myofibroma as well as a number of other entities, including infantile hemangiopericytoma, angioleiomyoma, and glomus tumor [5]. Desmoid tumor is easily excluded by immunophenotyping.

A small subset of patients with early-onset infantile myofibromatosis with multiorgan involvement died of disease, while adult-onset, intraosseous myofibromas are benign and rarely recur following surgical removal of the tumor, similar to their soft tissue counterpart.

In summary, we present an extraordinary rare case of adultonset, solitary, intraosseous myofibroma involving the middle phalanx of the index finger. To our knowledge, this was the 5 th adult case of myofibroma arising in non-craniofacial bones and the first reported case in the bones of hands and feet. Review of literature revealed that the adult-onset, intraosseous myofibromas behave distinctly different from the early-onset tumors, similar to their soft tissue counterpart in adults. Thus, awareness of this uncommon tumor in the adult patients is essential to prevent mistaken diagnosis and subsequent extensive workup.

Conflict of interest Yihong Ma declares that she has no conflict of interest.

Gene P. Siegal declares that he has no conflict of interest.

Shi Wei declares that he has no conflict of interest.

Statement of human and animal rights There is no violation of human and animal rights.

Statement of informed consent Informed consent was obtained.

\section{References}

1. Stout AP. Juvenile fibromatoses. Cancer. 1954;7:953-78.
2. Inwards CY, Unni KK, Beabout JW, et al. Solitary congenital fibromatosis (infantile myofibromatosis) of bone. Am J Surg Pathol. 1991;15:935-41.

3. Oudijk L, den Bakker MA, Hop WC, et al. Solitary, multifocal and generalized myofibromas: clinicopathological and immunohistochemical features of 114 cases. Histopathology. 2012;60:E1-11.

4. Smith KJ, Skelton HG, Barrett TL, et al. Cutaneous myofibroma. Mod Pathol. 1989;2:603-9.

5. Fletcher CDM, World Health Organization., International Agency for Research on Cancer. WHO classification of tumours of soft tissue and bone. 4th ed. IARC Press: Lyon, 2013

6. Goldblum JR, Folpe AL, Weiss SW, et al. Enzinger and Weiss's soft tissue tumors. 6th ed. Philadelphia, PA: Saunders/Elsevier; 2014.

7. Brunschweiler B, Guedj N, Lenoir T, et al. Oncogenous osteomalacia and myopericytoma of the thoracic spine: a case report. Spine. 2009;34:E857-860.

8. Asirvatham R, Moreau PG, Antonius JI. Solitary infantile myofibromatosis of axis. A case report. Spine. 1994;19:80-2.

9. Imaizumi S, Ogose A, Hotta T, et al. Solitary infantile myofibromatosis involving the clavicle. Skeletal Radiol. 1999;28: 473-6.

10. Oliver RJ, Coulthard P, Carre C, et al. Solitary adult myofibroma of the mandible simulating an odontogenic cyst. Oral Oncol. 2003;39: 626-9.

11. Sedghizadeh PP, Allen CM, Kalmar JR, et al. Solitary central myofibroma presenting in the gnathic region. Ann Diagn Pathol. 2004;8:284-9.

12. Bodkin PA, Choksey MS, Fagan J. Solitary calvarial myofibroma presenting in adolescence. Br J Neurosurg. 2005;19:420-4.

13. Konishi E, Mazaki T, Urata Y, et al. Solitary myofibroma of the lumbar vertebra: adult case. Skeletal Radiol. 2007;36 Suppl 1:S8690.

14. Biglioli F, Colombo V, Valassina D, et al. Miniretromandibular access for mandibular condyle biopsies. Minerva Stomatol. 2010;59:603-9.

15. Ramadorai A, Rajsekaran A, Narayanan V. A case report of solitary, intraosseous, adult-onset myofibroma of the mandible. J Maxillofac Oral Surg. 2010;9:280-3.

16. Brierley DJ, Khurram SA, Speight PM. Solitary myofibroma of the adult mandible: a case report. Oral Surg Oral Med Oral Pathol Oral Radiol. 2013;115:e40-43.

17. Sundaravel S, Anuthama K, Prasad H, et al. Intraosseous myofibroma of mandible: a rarity of jaws: with clinical, radiological, histopathological and immunohistochemical features. J Oral Maxillofac Pathol. 2013;17:121-5. 